\title{
Keratograph 5M As A Useful And Objective Tool For Evaluating The Ocular Surface In Limbal Stem Cell Deficiency
}

This article was published in the following Dove Press journal: Clinical Ophthalmology

\author{
Asunción Alfaro-Juárez (D) ${ }^{1, *}$ \\ Manuel Caro-Magdaleno $\mathbb{D}^{1,2, *}$ \\ Jesús Montero-Iruzubieta ${ }^{1,2}$ \\ Ana Fernández-Palacín (1D ${ }^{3}$ \\ Ana Muñoz-Morales (iD) \\ Manuel Alberto \\ Castilla-Martino' \\ Consuelo Spínola-Muñoz' \\ Enrique Rodríguez de la \\ Rúa ${ }^{1,2}$ \\ 'U.G.C. Oftalmología, Hospitales \\ Universitarios Virgen Macarena y Virgen \\ del Rocío, Sevilla, Spain; ${ }^{2}$ Deparmento de \\ Cirugía, Universidad de Sevilla, Sevilla, \\ Spain; ${ }^{3}$ Deparmento de Medicina \\ Preventiva y Salud Pública, Universidad de \\ Sevilla, Sevilla, Spain
}

*These authors contributed equally to this work

\begin{abstract}
Purpose: In limbal stem cell deficiency, both the Ocular Surface Disease Index (OSDI) questionnaire and tear break-up time (BUT) are comparable between traditional methods and the Keratograph $5 \mathrm{M}$. In this study, we aimed to correlate OSDI with Keratograph 5M interviewed OSDI, as well as slit-lamp tear BUT with Keratograph 5M noninvasive tear break-up time (NIKBUT) in limbal stem cell deficiency.
\end{abstract}

Patients and methods: Thirty-eight limbal stem cell-deficiency patients (76 eyes) from Virgen Macarena-Rocio Hospital (Seville, Spain) underwent this diagnostic test study. All patients completed the traditional OSDI. We measured the BUT, performed a Keratograph $5 \mathrm{M}$ analysis of NIKBUT first (employed for the analysis) followed by the average NIKBUT, the level of dryness, and conducted the OSDI questionnaire through an interview. For each pair of tests, we analyzed the means and applied an intraclass correlation coefficient $(r)$, creating a Bland-Altman plot for data dispersion.

Results: Average values were 47.5 points $( \pm 25.8)$, and 47.3 points $( \pm 27.5)$ for traditional OSDI and Keratograph OSDI, respectively $(P=0.87)$; the $r$ value indicates good agreement (0.72). The Bland-Altman plot followed a linear pattern, and the results were similarly distributed. The NIKBUT mean was shorter than the BUT mean $(P=0.007)$; the $r$ value indicates moderate agreement (0.574). The Bland-Altman plot formed an almost horizontal line, with almost all values between the mean and two standard deviations.

Conclusion: Keratograph $5 \mathrm{M}$ is useful for the evaluation of the ocular surface in limbal stem cell deficiency. NIKBUT can substitute BUT based on its advantages of being noninvasive, objective, with intraobserver and interobserver repeatability and reliability. The Keratograph 5M OSDI is comparable to the traditional questionnaire.

Keywords: keratograph, limbal stem cell deficiency, noninvasive keratograph tear break-up time, ocular surface desease index, tear break-up time

\section{Introduction}

The proper diagnosis of ocular surface diseases requires the recording of a patient's symptoms and the measurement of signs through various tests. The Ocular Surface Disease Index (OSDI) is a 12-item questionnaire that records the symptoms secondary to ocular surface problems and their impact on the patient's quality of life. The questionnaire is validated and widely used in daily clinical practice and research studies, thus enabling the comparison of results from different studies. The questionnaire has good validity, reliability, sensitivity, and specificity. ${ }^{1}$ The Keratograph 5M includes an OSDI, unlike the traditional OSDI, which is performed
Correspondence: Manuel

Caro-Magdaleno

U.G.C. Oftalmología, Hospitales

Universitarios Virgen Macarena y Virgen

del Rocío, C/Bogota 7, Bloq.3, Ist-B,

Sevilla 4I0I3, Spain

Tel +34609030403

Email mcaro79@gmail.com 
by a trained interviewer rather than being self-administered. On the other hand, the tear break-up time (BUT) is one of the most widely used tests, and it is useful for diagnosis and for managing the treatment of dry eyes. ${ }^{2}$ However, the test is invasive and observer-dependent, which gives it a low reproducibility and clear limitations when comparing results from different diseases and the response to treatments. ${ }^{3}$ There is, therefore, considerable interest in finding a method that can measure tear stability objectively, reproducibly, and noninvasively. The Tear Film and Ocular Surface Society Dry Eye Workshop (TFOS DEWS II $)^{4}$ recently recommended using automated noninvasive measurement techniques that allow for an objective assessment of the undisturbed tear film. The noninvasive BUT is performed noninvasively without fluorescein. The noninvasive BUT was primarily measured with the Tearscope (Keeler, Windsor, UK) which measures the break-up of tears by observing the distortion of an image projected on the cornea and counting the time it takes the image to break-up after a blink. The method is subjective; an observer uses the slit-lamp to observe any disruption in the tear image projected by the Tearscope. ${ }^{5}$ The noninvasive BUT test performed by the Keratograph $5 \mathrm{M}$ measures BUT based on the analysis of the disruption of the reflections of Placido rings in the tear film in an automated manner and without using staining, which gives the test the properties mentioned above.

One of our study objectives was to determine whether the difference in collecting information, i.e., between selfadministered tests and those that are conducted by trained interviewers, influences the test results. The comparison of both tear BUT measurements was the other goal of our study. Our target population was patients with limbal stem cell deficiency, due to the important surface problems they present and in which we should not use invasive techniques. To our knowledge, none of these comparisons have been studied in limbal stem cell deficiency. If the measurements are comparable, the use of Keratograph $5 \mathrm{M}$ in the evaluation of patients with limbal stem cell deficiency may be advantageous given the non-invasiveness of the technique on an already damaged surface.

\section{Materials And Methods}

This was a concordance study of additional tests and an assessment of diagnostic scales. The study was conducted with patients diagnosed with limbal stem cell deficiency from the Ophthalmology Clinical Management Unit at the University Hospital Virgen Macarena and the University
Hospital Virgen del Rocio of Seville. The inclusion criteria were patients who were older than 18 years old and signs and symptoms compatible with limbal stem cell deficiency according to the Dua clinical criteria. ${ }^{6}$ The exclusion criteria were patients who could not complete the case history review and basic clinical examination. The study was approved by the Ethics Committee of Hospital Virgen Macarena with a commitment to respect the fundamental principles of the Declaration of Helsinki and the Council of Europe Convention regarding human rights and biomedicine. The patients were informed of the details of the study and given an information sheet and an informed consent form, which they signed in duplicate. The data recorded during the study are protected by Law 15/1999 of December 13 on the Protection of Personal Data.

To calculate the sample size, we employed nQuery Advisor Release 7.0 (Statistical Solutions, Broadway, Saugus, Massachusetts, USA, 2002). We employed the intraclass correlation coefficient as a measure of the reliability between measurements performed by different methods on the same participants. To this end, we calculated a sample size for which we assumed an expected value of 0.6 in the most unfavorable situation, a 95\% confidence level, an interval precision or amplitude of 0.15 and the performance of two measurement methods. With this information, we calculated a sample size of at least 71 eyes.

In the initial visit, we recorded age, sex, and etiology of the limbal stem cell deficiency and performed the traditional OSDI questionnaire and a slit-lamp examination of the BUT. The OSDI employed was the Spanish-validated version. ${ }^{7}$ This is a self-administered questionnaire that was given to the patients who were instructed to fill it out. The resulting values classified dry eyes as "normal" for $0-12$, "mild" for 13-22, "moderate" for 23-32, and "severe" for $33-100 .{ }^{8}$ We performed the BUT once per eye after instilling in the inferior fornix one $2 \%$ fluorescein eye drop (Colircusí Fluoresceína, $20 \mathrm{mg} / \mathrm{mL}$, colirio en solución, Alcon-Cusí, El Masnou, Barcelona, España) and waiting for the break-up of the tear film. No micropipette was used because they were not available. We employed the slitlamp's blue light with the beam width fully open. Values $\leq 10$ seconds were considered pathological.

In order to prevent alteration of the keratograph results, the two visits were separated by at least $48 \mathrm{hrs}$ from the time when fluorescein eye drops were instilled. The same room with the same lighting (mesopic conditions) and the temperature was used for all encounters. Temperature and 
humidity in the room were measured and registered and ranged between $22-25^{\circ} \mathrm{C}$ and $45-55 \%$, respectively.

During the second visit, we performed a study of the ocular surface using the Keratograph 5M (Oculus, Wetzlar, Germany). Using the Oculus TF-Scan module, we measured the Keratograph 5M noninvasive tear BUT (NIKBUT) (once per eye), which is a noninvasive measure of the time between a blink and the disruption of the rings reflected in the tear film. The Keratograph $5 \mathrm{M}$ provides the first and average NIKBUT and the level of dry eye according to the tear BUT. Of these, we mainly used the NIKBUT-first because it more closely resembles the original BUT. After the examination with the TF-Scan, we performed the OSDI questionnaire, which is included in the Keratograph $5 \mathrm{M}$. The questionnaire was administered by the examiner during the patient interview.

Data analysis was performed using IBM SPSS Statistics $22.0 \bigodot$ statistical suite for Windows $@$. In the descriptive analysis, we used percentages for the qualitative variables and means for the quantitative variables (and standard deviations) and medians (with P25 and P75 percentiles for markedly asymmetric distributions. In the study of the correlation between traditional OSDI and Keratograph OSDI and between BUT and NIKBUT, we analyzed the means for any differences. After confirming that there were no statistically significant differences, we applied an intraclass correlation coefficient $(r)$. To observe the data dispersion, we generated a BlandAltman plot.

\section{Results}

We recruited 38 patients (76 eyes), with a mean age of 54.63 years (95\% CI: 34.73-74.53). Of all the patients, $57.1 \%$ were women. The etiology is presented in Figure 1.

The traditional OSDI in hardcopy format achieved an average value of 47.5 points $( \pm 25.8)$, while the

\section{ETIOLOGY}

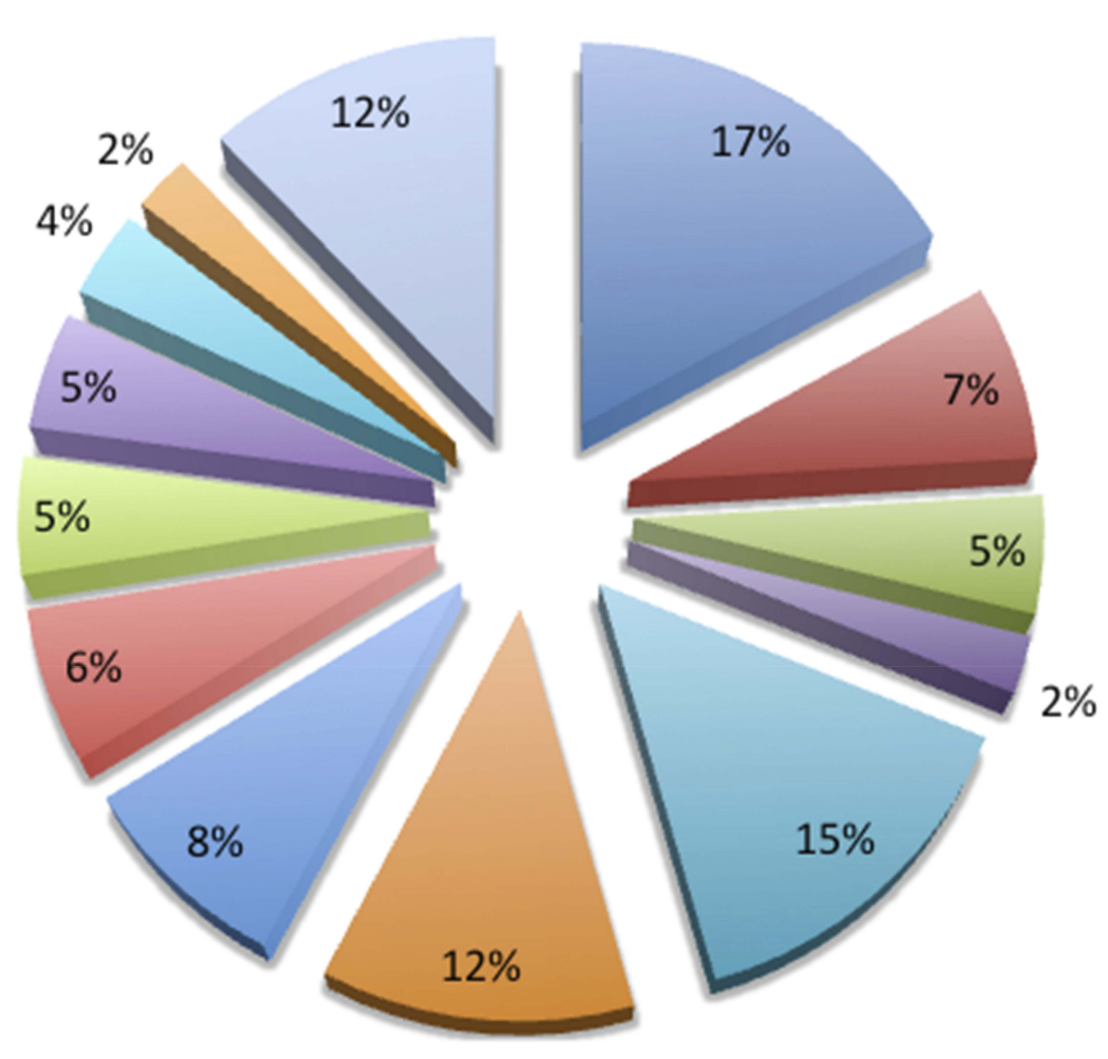

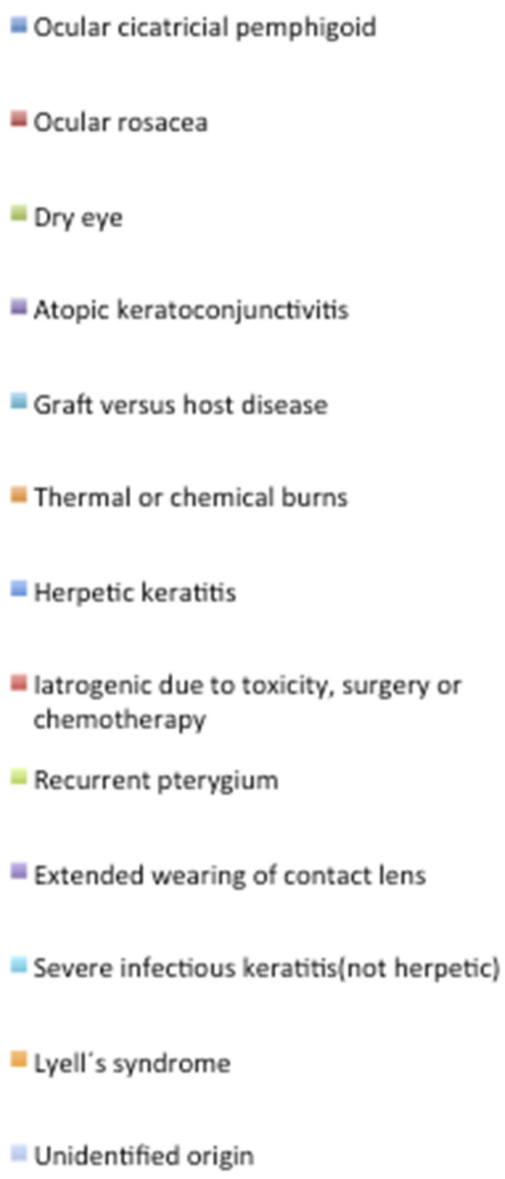

Unidentified origin

Figure I Sector plot of the etiology of limbal stem cell deficiency. The corresponding percentages for each group are shown. Each etiology is represented with a different color. 
Keratograph OSDI scored 47.3 points $( \pm 27.5)$. There were no statistically significant differences $(P=0.87)$ between the two means. The intraclass correlation coefficient achieved a value of $0.72(0.53-0.834)$, which indicates a good agreement between the two questionnaires. In the Bland-Altman plot, we observed that the measures were encompassed between the mean and two standard deviations following a linear pattern, demonstrating that the results of the two tests were very similar. See the plot in Figure 2.

Concerning tear break-up time, we observed a bias since our measures of BUT were limited to 10 seconds, the borderline value of normality, while the NIKBUT automatically reached 24 seconds as a maximum value. For comparison purposes, the data were adjusted, and we selected the NIKBUT data with values $\leq 10$ seconds. The mean BUT and the NIKBUT-first values were 3.3 seconds (95\% CI: $0.3-6.3)$ and 2.46 seconds (95\% CI: -0.64 to +5.56$)$, respectively. When comparing the mean BUT with the NIKBUT-first values, we obtained statistically significant differences $(p=0.007)$. The NIKBUTfirst values were shorter than the BUTvalues (difference ranging from 0.97 to 4.8 ). From here, we analyzed the intraclass correlation coefficient, obtaining a value of $0.574(0.213-0.769)$ which was considered moderate agreement (0.4-0.6) between the two tests. In the Bland-Altman plot, we observed that the NIKBUT and BUT measures fit one another, forming an almost horizontal line, showing linearity between the two tests that measure the same variable but with different methods. Almost all values were between the mean and two standard deviations (Figure 3). We also observed that the greatest dispersion was for the nonextreme values in the middle area of the graph.

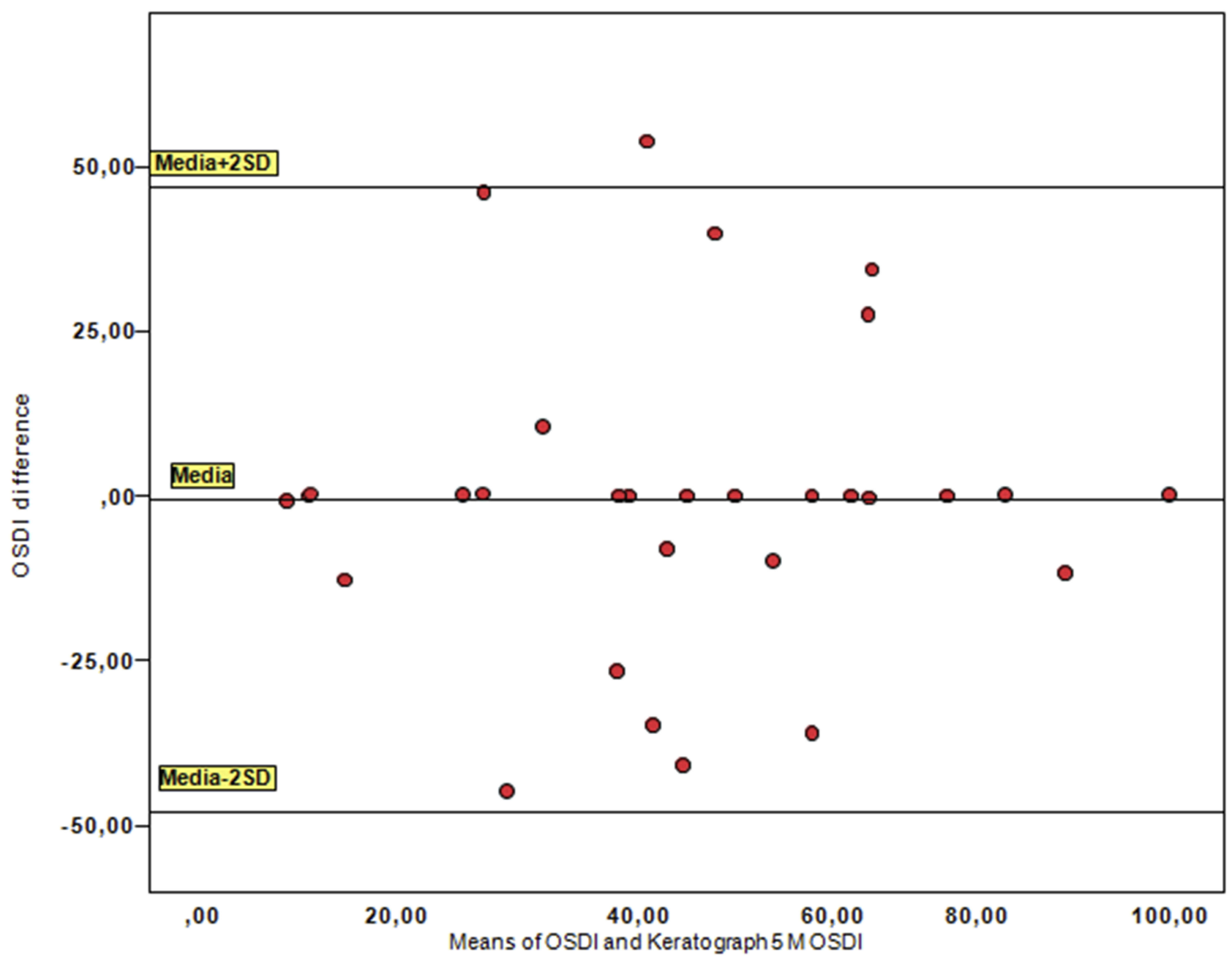

Figure 2 Bland-Altman plot of the relationship between the classical self-administered Ocular Surface Disease Index (OSDI) and the Keratograph 5M OSDI. The measures were encompassed between the mean and two standard deviations following a linear pattern, demonstrating that the results of the two tests were very similar. 


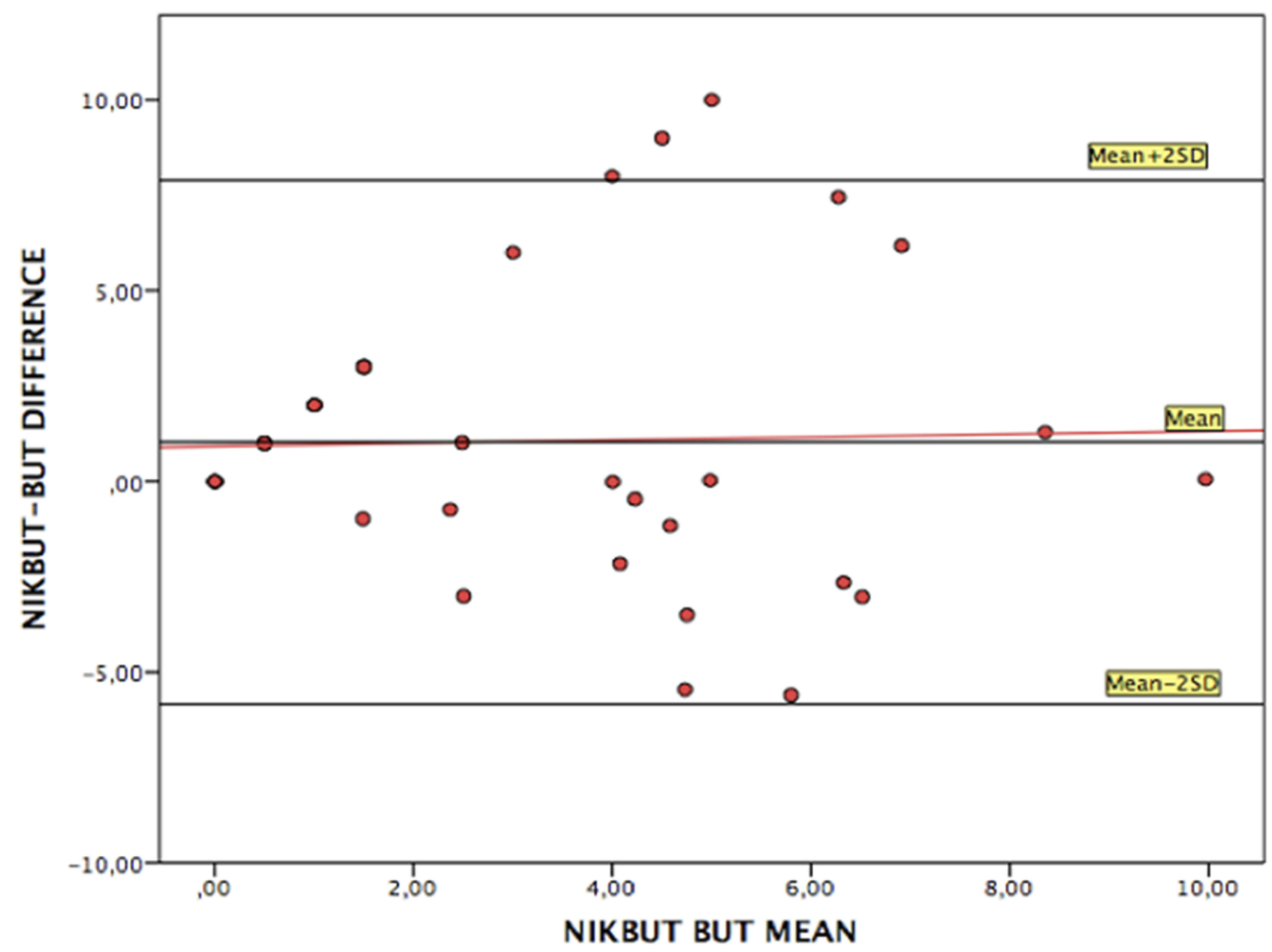

Figure 3 Bland-Altman plot of the relationship between the keratograph noninvasive tear break-up time (NIKBUT) and tear break-up time (BUT). The two tests fit one another, forming an almost horizontal line, showing linearity. Almost all values were between the mean and two standard deviations.

\section{Discussion}

We first analyzed the correlation between the traditional OSDI translated and validated for Spanish and the interviewed Keratograph OSDI. The OSDI questionnaire validated by Rhett Schiffman et al in $2000^{1}$ was designed for diagnosing any type of dry eyes and classifying its severity. The questionnaire had good correlations with other tests for dry eyes such as the Schirmer and tear BUT tests, the 25-item National Eye Institute Visual Function Questionnaire, McMonnies, and the Short Form-12 Health Status questionnaires. ${ }^{1,9}$ Although the OSDI was originally designed for dry eye disease, it has also been used for assessing the effect on the ocular surface of antiglaucoma treatment, ${ }^{10}$ contact lens, ${ }^{11}$ thyroid eye disease, ${ }^{12}$ and Meibomian gland dysfunction, ${ }^{13}$ among other numerous diseases. The 2007 Dry Eye WorkShop workgroup described the OSDI questionnaire. ${ }^{14}$ It should be noted that the OSDI appears as a self-completion test where the patient is invited to fill out its 12 questions. The text of the Keratograph OSDI is similar to that of the traditional OSDI; however, the method for collecting the information is substantially different because for the Keratograph $5 \mathrm{M}$ the examiner asks the questions and can answer the patient's doubts in a dynamic and interactive setting. Based on our results, we can confirm that although both questionnaires change their method for collecting the information, they use similar methods for measuring ocular surface symptoms, at least in patients with limbal stem cell deficiency, with either of the two questionnaires being used interchangeably. Clayton et al studied the correlation of several questionnaires used for measuring vision, comparing the classical hardcopy questionnaires and those filled in via webpages, for patients with ocular surface disease $(n=68)$ and healthy patients $(n=50)$. Similar to our experience with the OSDI questionnaire, the authors found no statistically significant differences between the 
two methods. The only statistically significant result was among the scores achieved by patients older than 40 years, which was higher than that of patients younger than 40 years, in both types of questionnaires and due to poorer ocular surface condition ${ }^{15}$ OSDI was designed to analyze dry eye disease and severe dry eye was considered when OSDI score was greater than 33. Our patients presented a higher rate of ocular discomfort, with a mean score of 47 points due to the fact that limbal stem cell deficiency is related to serious ocular surface problems. ${ }^{8}$

We correlated the BUT and the NIKBUT. Both these parameters measure the same variable (tear film stability) but in different ways. A BUT $<10$ seconds is abnormal, ${ }^{16}$ and a BUT $<5$ seconds indicates dry eyes, ${ }^{14}$ while a BUT between 5 and 10 seconds is marginal or suspicious. According to Goto et al, BUT has a sensitivity and specificity for dry eyes of $75 \%$ and $60 \%$, respectively. ${ }^{17}$ However, BUT is known to have low reproducibility, because this test is influenced by the examiner's experience, partial blinking, the type of slit-lamp lighting and by the characteristics of the fluorescein eye drops (volume instilled, $\mathrm{pH}$, concentration, preservatives, fluorescein strips versus eye drops). ${ }^{3,18,19}$ There have, therefore, been attempts to measure tear stability objectively, reproducibly, and noninvasively. ${ }^{5}$

The NIKBUT is considered a noninvasive ("does not involve instillation of fluorescein, blinking is natural, not forced or suppressed and there is no contact between the measuring instrument and the eye or eyelids"5) and objective method that measures BUT based on the analysis of disruption of the reflections of Placido rings in the tear film. The Keratograph $5 \mathrm{M}$ provides several measures of this parameter: the NIKBUT-first, the average NIKBUTand the level of dry eyes according to the NIKBUT. NIKBUT-first refers to the tear BUT that first occurs after a blink and the disruption of one of the rings projected on the tear film. The average NIKBUT is the mean break-up time of all measured areas in the cornea within 24 seconds, which is the maximum measurement period. The level of dry eyes according to the NIKBUT is the level of tear instability the software assigns according to the reference values in Table 1 (Keratograph 5M dry eye level).

In 1985, Mengher obtained higher values with noninvasive BUT than with BUT in healthy patients and rationalized these results by the tear instability caused by the fluorescein eye drops. ${ }^{16}$ Cebreiro et al reported that the automatic noninvasive BUT measurements (e.g., NIKBUT) were similar to those obtained clinically (BUT) by an expert observer. ${ }^{20}$
Table I Keratograph 5M Dry Eye Level

\begin{tabular}{|l|l|l|}
\hline Dry Eye Level & $\begin{array}{l}\text { First Keratograph } \\
\text { 5M Noninvasive } \\
\text { Tear Break-Up } \\
\text { Time }\end{array}$ & $\begin{array}{l}\text { Mean } \\
\text { Keratograph 5M } \\
\text { Noninvasive Tear } \\
\text { Break-Up Time }\end{array}$ \\
\hline $\begin{array}{l}\text { Stable } \\
\text { Suspected }\end{array}$ & $\begin{array}{l}\geq 10 \text { seconds } \\
\text { Abnormal } \\
\text { "Too short of a } \\
\text { time" }\end{array}$ & $\begin{array}{l}\leq 5 \text { seconds } \\
\leq 1.5 \text { seconds }\end{array}$ \\
\hline
\end{tabular}

Other authors such as Cho, Nichols et al obtained poor correlations between BUT and noninvasive BUT (not automated like the NIKBUT), with typically longer noninvasive BUT results than the BUT. ${ }^{21,22}$ In 2011, Pult et al compared tear stability, measured with the Keeler Tearscope (nonautomated noninvasive BUT) and Oculus Keratograph (NIKBUT) and obtained a good correlation $(r=0.54)$. However, the NIKBUT was shorter than the noninvasive BUT obtained with the Tearscope. On the other hand, the authors observed better reproducibility and predictability for dry eyes (diagnosis based on symptoms and OSDI) with the Tearscope than with the Keratograph. ${ }^{23}$ Best et al also obtained shorter values with the Keratograph than with the Tearscope, observing a better correlation between the values obtained with the Tearscope and the patient's symptoms. These authors reported that when the Keratograph is adjusted to the Tearscope values, there is a better adjustment to the symptoms. $^{24}$ Hong and Best obtained shorter NIKBUT values than with the classical BUT and Tearscope, respectively. ${ }^{24,25}$ These authors postulated that, because it is a more objective and sensitive method, it can more quickly detect tear break-up than an observer in the slit-lamp. ${ }^{24,25}$ Moreover, Madden et al highlighted the lack of interobserver reproducibility in the noninvasive BUT when this was measured subjectively, as occurs with the Tearscope. ${ }^{26}$ Repeatability and reproducibility of NIKBUT obtained with Keratograph $5 \mathrm{M}$ were acceptable for both healthy and dry eye patients. ${ }^{27}$ In our study, the BUT and the NIKBUT measurements were performed by a single examiner, although the NIKBUT does not depend on the examiner.

Although our results confirm shorter times of the NIKBUT when compared with the BUT, the real problem with the noninvasive BUT (obtained with the Tearscope) and BUT is that the methods are subjective and lack reproducibility, as confirmed by Madden. ${ }^{26}$ The results of these methods cannot, therefore, be inferred and compared 
with those of other studies, treatments, or diseases. The NIKBUT is obtained objectively by the Keratograph and is observer-independent. The data cannot be modified, and greater accuracy is achieved than with subjective methods. In this study, the NIKBUT was obtained by following Mengher's specifications, who describes the NIKBUT as the time from the last complete blink to the first distortion of the rings projected on the tear film. ${ }^{16}$ Hong et al reported that the NIKBUT values were lower than the BUT values (measured with $1 \%$ fluorescein without preservatives) and stated that the Keratograph $5 \mathrm{M}$ detects tear film disruptions earlier, even though the two measures are well correlated (Spearman's test $P=0.55$ ). ${ }^{25}$ Our results are similar to those of Hong et al; the values obtained with the NIKBUT were shorter than those with BUT. Furthermore, Hong et al reported good intraobserver and interobserver reproducibility for the NIKBUT, with coefficients of variation $(\mathrm{CV})$ of $12.8 \%$ and $15.4 \%$, respectively, and intraclass correlation coefficients $(r)$ of 0.93 and 0.88 , respectively. ${ }^{25}$ In our study, the intraclass correlation coefficient was lower although moderate to good ( $r$ 0.57). We should clarify that Hong measured this parameter in a more uniform sample with dry eye disease, while we measured it in patients with limbal stem cell deficiency of multiple etiologies and in different stages, which could be a source of variability in this measure. Lastly, Hong et al obtained a sensitivity for dry eyes of $84.1 \%$ and a specificity of $75.6 \% .{ }^{25}$ Sweeney et al advocated methods for measuring tear stability that was objective and that only measured in the interval of a normal blink, without using any substance that could change the blink. These authors also indicated that the study of the lipid portion of the tear (e.g., through interferometry) could provide more information. ${ }^{5}$ The Keratograph $5 \mathrm{M}$ also measures the lipid layer of tears according to a color scale assisted by interferometry; however, the Keratograph $5 \mathrm{M}$ still does not quantify the layer numerically. These objectives methods for measuring tear stability should also have intraobserver and interobserver repeatability and reliability for comparing the results of patients with different diseases and in response to different treatments.

The current study had a few limitations. First, the inclusion of patients with limbal stem cell deficiency of multiple etiologies and in different stages results in a source of variability. Although it is difficult to recruit a proper sample size of this disease, more homogeneous samples must be recruited and only one eye per patient must be measured in further studies. Secondly, questionnaire administration was not randomized in this study. We recommend a randomization in the order of administration in future studies. Also, repeated measurements per eye and the use of a micropipette to control the amount of fluorescein instilled could improve the data.

\section{Conclusion}

The NIKBUT can thereby be an appropriate substitute for BUT, providing advantages of noninvasiveness, objectivity, intraobserver and interobserver repeatability, and reliability to the already damaged ocular surface of limbal stem celldeficient patients. Both tests are well correlated, even though the population had various degrees of limbal stem cell deficiency and different etiologies that affect the ocular surface condition. Also, the device includes an OSDI comparable to that of the traditional questionnaire. For these reasons, we propose that the Keratograph $5 \mathrm{M}$ is a useful tool for evaluating limbal stem cell deficiencysurface. Despite having used a considerable sample size given the entity we manage, the evidence provided by the results could be improved by increasing the sample for proper randomization. Further studies along this line are warranted.

\section{Acknowledgments}

Iniciativa Andaluza en Terapias Avanzadas (IATA). RETICS OFTARED "RD16/0008/0010", financed by the Instituto de Salud Carlos III, as part of the Plan Nacional $\mathrm{I}+\mathrm{D}+\mathrm{i}$, and co-financed by the European Union (FEDER/ FSE) "Una manera de hacer Europa2013-2016".

\section{Disclosure}

Iniciativa Andaluza en Terapias Avanzadas (IATA) and OFTARED "RD16/0008/0010" financed the translation of the article. The authors report no other conflicts of interest in this work.

\section{References}

1. Schiffman RM, Christianson MD, Jacobsen G, Hirsch JD, Reis BL. Reliability and validity of the ocular surface disease index. Arch Ophthalmol. 2000;118:615-621. doi:10.1001/archopht.118.5.615

2. Nichols KK, Nichols JJ, Zadnik K. Frequency of dry eye diagnostic test procedures used in various modes of ophthalmic practice. Cornea. 2000;19:477-482. doi:10.1097/00003226-200007000-00015

3. Vanley GT, Leopold IH, Gregg TH. Interpretation of tear film breakup. Arch Ophthalmol. 1977;95:445-448. doi:10.1001/archopht.1977.0445 0030087010

4. Wolffsohn JS, Arita R, Chalmers R, et al. The ocular surface TFOS DEWS II diagnostic methodology report. Ocul Surf. 2017;15:539-574. doi:10.1016/j.jtos.2017.05.001

5. Sweeney DF, Millar TJ, Raju SR. Tear film stability: a review. Exp Eye Res. 2013;117:28-38. doi:10.1016/j.exer.2013.08.010 
6. HS D. Essentials in Ophthalmology. Cornea and External Eye Disease. ( $G K R N W, E d$.). Germany: Springer Berlin Heidelberg 2006.

7. Garcia-Catalan MR, Jerez-Olivera E, Benitez-Del-Castillo-Sanchez JM. Dry eye and quality of life. Arch Soc Esp Oftalmol. 2009;84: 451-458.

8. Amparo F, Schaumberg DA, Dana R. Comparison of two questionnaires for dry eye symptom assessment: the ocular surface disease index and the symptom assessment in dry eye. Ophthalmology. 2015;122:1498-1503. doi:10.1016/j.ophtha.2015.02.037

9. Vitale S, Goodman LA, Reed GF, Smith JA. Comparison of the NEIVFQ and OSDI questionnaires in patients with Sjögren's syndromerelated dry eye. Health Qual Life Outcomes. 2004;2:44. doi:10.1186/ 1477-7525-2-44

10. Garcia-Feijoo J, Sampaolesi J. A multicenter evaluation of ocular surface disease prevalence in patients with glaucoma. Clin Ophthalmol. 2012;6:441-446.

11. Theophanous C, Irvine JA, Parker P, Chiu GB. Use of prosthetic replacement of the ocular surface ecosystem scleral lenses in patients with ocular chronic graft-versus-host disease. Biol Blood Marrow Transplant. 2015;21:2180-2184. doi:10.1016/j.bbmt.2015.06.012

12. Kocabeyoglu S, Mocan MC, Cevik Y, Irkec M. Ocular surface alterations and in vivo confocal microscopic features of corneas in patients with newly diagnosed graves' disease. Cornea. 2015;34: 745-749. doi:10.1097/ICO.0000000000000426

13. Yao W, Liang Q, Sun X, Wang N, Antoine L. Evaluation of the diagnostic value for meibomian gland dysfunction examinations. Chinese J Ophthalmol. 2014;50:247-253.

14. Gipson IK, Argüeso P, Beuerman R, et al. Research in dry eye: report of the research subcommittee of the international dry eye workshop (2007). Ocul Surf. 2007;5:179-193. doi:10.1016/S1542-0124(12)70086-1

15. Clayton JA, Eydelman M, Vitale S, Manukyan Z, Kramm R, Datiles M 3rd. Web-based versus paper administration of common ophthalmic questionnaires: comparison of subscale scores. Ophthalmology. 2013;120:2151-2159. doi:10.1016/j.ophtha.2013.03.019

16. Mengher LS, Bron AJ, Tonge SR, Gilbert DJ. Effect of fluorescein instillation on the pre-corneal tear film stability. Curr Eye Res. 1985;4:9-12. doi:10.3109/02713688508999961
17. Goto T, Zheng X, Okamoto S, Ohashi Y. Tear film stability analysis system: introducing a new application for videokeratography. Cornea. 2004;23:S65-S70. doi:10.1097/01.ico.00001366 85.88489 .70

18. Mengher LS, Bron AJ, Tonge SR, Gilbert DJ. A non-invasive instrument for clinical assessment of the pre- corneal tear film stability. Curr Eye Res. 1985;4:1-7. doi:10.3109/02713688508999960

19. Cho P, Brown B, Chan I, Conway R, Yap M. Reliability of the tear break-up time technique of assessing tear stability and the locations of the tear break-up in Hong Kong Chinese. Optom Vis Sci. 1992;69:879-885. doi:10.1097/00006324-199211000-00007

20. Cebreiro E, Ramos L, Mosquera A, Barreira N, Penedo MF. Automation of the tear film break-up time test. Eur J Ophthalmol. 2012;22:S17-S23.

21. Cho P, Douthwaite W. The relation between invasive and noninvasive tear break-up time. Optom Vis Sci. 1995;72:17-22. doi:10.1097/ 00006324-199504000-00003

22. Nichols JJ, Nichols KK, Puent B, Saracino M, Mitchell GL. Evaluation of tear film interference patterns and measures of tear break-up time. Optom \& Vis. 2002;79:363-369. doi:10.1097/000063 24-200206000-00009

23. Pult H, Riede-Pult B. The new TF-scan application for video keratography: its repeatability and usefulness in tear film break-up time analysis. Contact Lens Anterior Eye. 2011;34:S14. doi:10.1016/S13 67-0484(11)60073-8

24. Best N, Drury L, Wolffsohn JS. Clinical evaluation of the oculus keratograph. Contact Lens Anterior Eye. 2012;35:171-174. doi:10.10 16/j.clae.2012.04.002

25. Hong J, Sun X, Wei A, et al. Assessment of tear film stability in dry eye with a newly developed keratograph. Cornea. 2013;32:716-721. doi:10.1097/ICO.0b013e3182714425

26. Madden RK, Paugh JR, Wang C. Comparative study of two noninvasive tear film stability techniques. Curr Eye Res. 1994;13:263269. doi: $10.3109 / 02713689408995787$

27. Tian L, Qu JH, Zhang XY, Sun XG. Repeatability and reproducibility of noninvasive keratograph $5 \mathrm{M}$ measurements in patients with dry eye disease. J Ophthalmol. 2016;2016:8013621. 


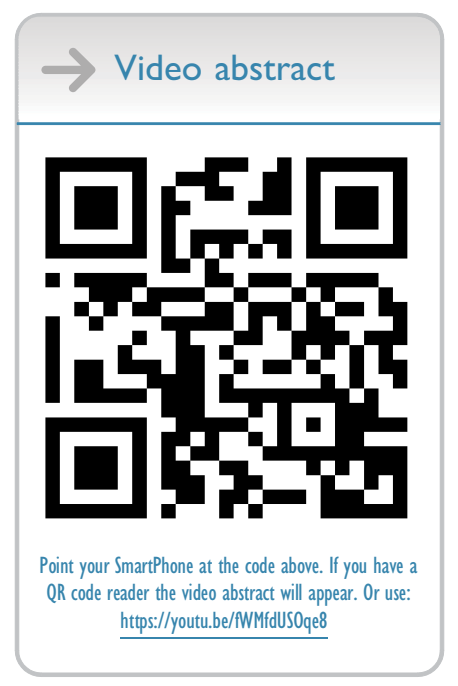

Clinical Ophthalmology

\section{Publish your work in this journal}

Clinical Ophthalmology is an international, peer-reviewed journal covering all subspecialties within ophthalmology. Key topics include: Optometry; Visual science; Pharmacology and drug therapy in eye diseases; Basic Sciences; Primary and Secondary eye care; Patient Safety and Quality of Care Improvements. This journal is indexed on PubMed

Submit your manuscript here: https://www.dovepress.com/clinical-ophthalmology-journal
Central and CAS, and is the official journal of The Society of Clinical Ophthalmology (SCO). The manuscript management system is completely online and includes a very quick and fair peer-review system, which is all easy to use. Visit http://www.dovepress.com/ testimonials.php to read real quotes from published authors. 